\title{
On the origin of the high helium sequence in $\omega$ Centauri
}

\author{
A. Maeder and G. Meynet
}

Geneva Observatory, 1290 Sauverny, Switzerland

e-mail: [andre.maeder; georges. meynet] @obs . unige.ch

Received 19 December 2005 / Accepted 21 January 2006

\section{ABSTRACT}

The blue Main Sequence (bMS) of $\omega$ Cen implies a ratio of helium to metal enrichment $\Delta Y / \Delta Z \approx 70$, which is a major enigma. We show that rotating models of low metallicity stars, which account for the anomalous abundance ratios of extremely metal poor stars, are also useful for understanding the very high $\Delta Y / \Delta Z$ ratio in $\omega$ Cen. Models of massive stars with moderate initial rotation velocities produce stellar winds with large He- and $\mathrm{N}$-excesses, but without the large $\mathrm{C}$ - (and $\mathrm{O}-$ ) excesses made by very fast rotation, in agreement with the observed chemical abundance ratios in $\omega$ Cen.

It is still uncertain whether the abundance peculiarities of $\omega$ Cen result from the fact that the high velocity contributions of supernovae escaped the globular cluster, usually considered as a tidally stripped core of a dwarf galaxy. Another possibility is a general dominance of wind ejecta at very low $Z$, due to the formation of black holes. Some abundance and isotopic ratios like $\mathrm{Mg} / \mathrm{Al}, \mathrm{Na} / \mathrm{Mg}, \mathrm{Ne} / \mathrm{N},{ }^{12} \mathrm{C} /{ }^{13} \mathrm{C},{ }^{16} \mathrm{O} /{ }^{18} \mathrm{O}$ and ${ }^{17} \mathrm{O} /{ }^{18} \mathrm{O}$ may allow us to further discriminate between these scenarios and between the AGB and massive star contributions.

Key words. stars: $\omega$ Centauri - helium - stars: evolution

\section{Introduction}

The globular cluster $\omega$ Centauri has remarkable properties: it is the most massive globular cluster in the Galaxy and is often interpreted as the remaining core of an ancient dwarf galaxy (Bekki \& Freeman 2003), a possibility supported by the density profile of the cluster (Ideta \& Makino 2004). Dynamical studies support a formation of $\omega$ Cen from one of the small progenitor galaxies of the Milky Way (e.g. Gnedin et al. 2002).

The stars in $\omega$ Cen show a wide spread in metallicity (Norris \& Da Costa 1995) from $[\mathrm{Fe} / \mathrm{H}]=-2$ to -0.5 . Among its many abundance peculiarities, $\omega$ Cen shows a large $\mathrm{N}$-excess, an overabundance of s-elements relatively to $\mathrm{Fe}$ (Norris \& Da Costa 1995) and an unusually low $[\mathrm{Cu} / \mathrm{Fe}]$ ratio relatively to other metal poor stars (Cunha et al. 2002; McWilliam \& Smecker-Hane 2005), which is interpreted as a relative lack of contributions from supernovae SNIa (Cunha et al. 2002).

The finding of a double sequence in the globular cluster $\omega$ Cen by Anderson (1997; see also Bedin et al. 2004; Gratton 2005) and the further interpretation of the bluer sequence by a strong excess of helium constitutes a major enigma for stellar and galactic evolution (Norris 2004). The interpretation in terms of an He excess is convincing and supported by stellar models as well by the morphology of the horizontal branch stars (Piotto et al. 2005). The great problem is that the bluer sequence with a metallicity $[\mathrm{Fe} / \mathrm{H}]=-1.2$ or $Z=2 \times 10^{-3}$ implies an He-content $Y=0.38(0.35-0.45)$, i.e. an He-enrichment $\Delta Y=0.14$ (see Norris 2004). In turn, this demands a relative helium to metal enrichment $\Delta Y / \Delta Z$ of the order of 70 (Piotto et al. 2005; Gratton 2005). At the opposite, the system of globular clusters has a constant $Y=0.250$ (Salaris et al. 2004), while $[\mathrm{Fe} / \mathrm{H}]$ varies a lot, which implies a ratio $\Delta Y / \Delta X(\mathrm{Fe})=0$, where $X(\mathrm{Fe})$ is the iron mass fraction. The value $\Delta Y / \Delta Z=70$ is enormous and more than one order of magnitude larger than the current value of $\Delta Y / \Delta Z=4-5$ (Pagel et al. 1992) obtained from extragalactic HII regions. A value of 4-5 is consistent with the chemical yields from supernovae (Maeder 1992) forming black holes above about 20-25 $M_{\odot}$.

The subject of the present work is to examine whether this extreme value of $\Delta Y / \Delta Z$ can be accounted for by models of rotating stars at very low metallicity. These models, which have the same physical ingredients as the models successfully used at solar Z, well account (Meynet et al. 2006) for the abundance anomalies observed in extremely metal poor halo stars.

Section 2 collects the relevant observational determinations of the chemical abundances in $\omega$ Cen and mentions the possible interpretations of the observed abundance peculiarities. In Sect. 3, we show results of models of rotating stars and in Sect. 4 we compare the results to observations of the bMS in $\omega$ Cen. Section 5 gives the conclusions.

\section{The chemical abundances of the blue Main Sequence (bMS) in $\omega$ Cen}

Let us quote the anomalies related to the blue Main Sequence according to Piotto et al. (2005):

- The bMS is best supported by a strong helium enhancement (Norris 2004). A metallicity $Z=2 \times 10^{-3}$ with a helium content $Y=0.38$ is obtained by Piotto et al. (2005) 
by comparing observed spectra with synthetic spectra and adjusting the stellar structure models. For the red Main Sequence (rMS), these values are $Z=10^{-3}$ and $Y=0.246$. Thus, the $\Delta Y / \Delta Z$ ratio corresponding to the difference between the rMS and the bMS is $\sim 130$.

- The metallicity of the bMS is $[\mathrm{Fe} / \mathrm{H}]=-1.26$ compared to -1.57 for the rMS.

- The carbon to metal excess is $[\mathrm{C} / \mathrm{M}]=0$ for both the bMS and rMS.

- For nitrogen, Piotto et al. find $[\mathrm{N} / \mathrm{M}]=1.0-1.5$ for the bMS and $[\mathrm{N} / \mathrm{M}] \leq 1.0$ for the rMS.

- For s-elements, one has for example $[\mathrm{Ba} / \mathrm{M}]=0.7$ (with an excess $[\mathrm{Ba} / \mathrm{Eu}])$ for the bMS and 0.4 for the rMS.

- The scatter of the abundances of the bMS stars is small, while it is large for rMS'.

- From radial velocity and proper motions studies, there is no difference between stars on the rMS and bMS: both sequences belong to $\omega$ Cen (Piotto et al. 2005).

Various possible explanations of the peculiar helium content of the bMS stars have been mentioned by Norris (2004) and by Piotto et al. (2005). A possibility is galactic winds where the wind of high mass supernovae escaped the core of the dwarf galaxy, while the He-rich winds of lower mass SNII are kept in. The models with mixing and fallback (Umeda \& Nomoto 2003) offer a wide range of possibilities due to the internal freedom in the choice of parameters, however the agreement with observational constraints has not yet been demonstrated. Piotto et al. (2005) examine the possibility that the required helium is produced by AGB stars, however they note that the amount of helium produced seems insufficient. In addition, AGB models would also produce $\mathrm{C}$ efficiently and this is not observed. Norris (2004) proposed massive stars as the source of helium. However, the yields of massive stars, as mentioned above, usually lead to much lower $\Delta Y / \Delta Z$ ratios than the one necessary for $\omega$ Cen. Another possibility mentioned by Piotto et al. (2005) is that $\omega$ Cen was enriched by supernovae in the mass range of 10-14 $M_{\odot}$, which produce significant $\mathrm{He}$ as well as some $\mathrm{N}$ (Thielemann et al. 1996). The problem is, however, that such stars are not the favored source of s-element and it is hard to understand why the contributions of more massive stars are absent. Bekki \& Norris (2005) suggests that the bMs is formed from gas ejected from field stars that surrounded $\omega$ Cen, when it was a galaxy nucleus. However, the star models used to draw this conclusion do not account for rotationally enhanced mass loss.

\section{Rotating models of very low metallicity stars}

The main features of rotating low $Z$ star models are the following ones (Maeder \& Meynet 2001; Meynet et al. 2006):

- Low Z stars easily reach break-up during MS phase and thus lose mass, by the combined effect of stellar winds and rotation (cf. Maeder \& Meynet 2000a).

- Low $Z$ stars are compact and the external Gratton-Öpik, cell of meridional circulation is negligible due to the high stellar density. This leads to steep $\Omega$-gradients in both AGB and massive stars at very low $Z$, which favor mixing of

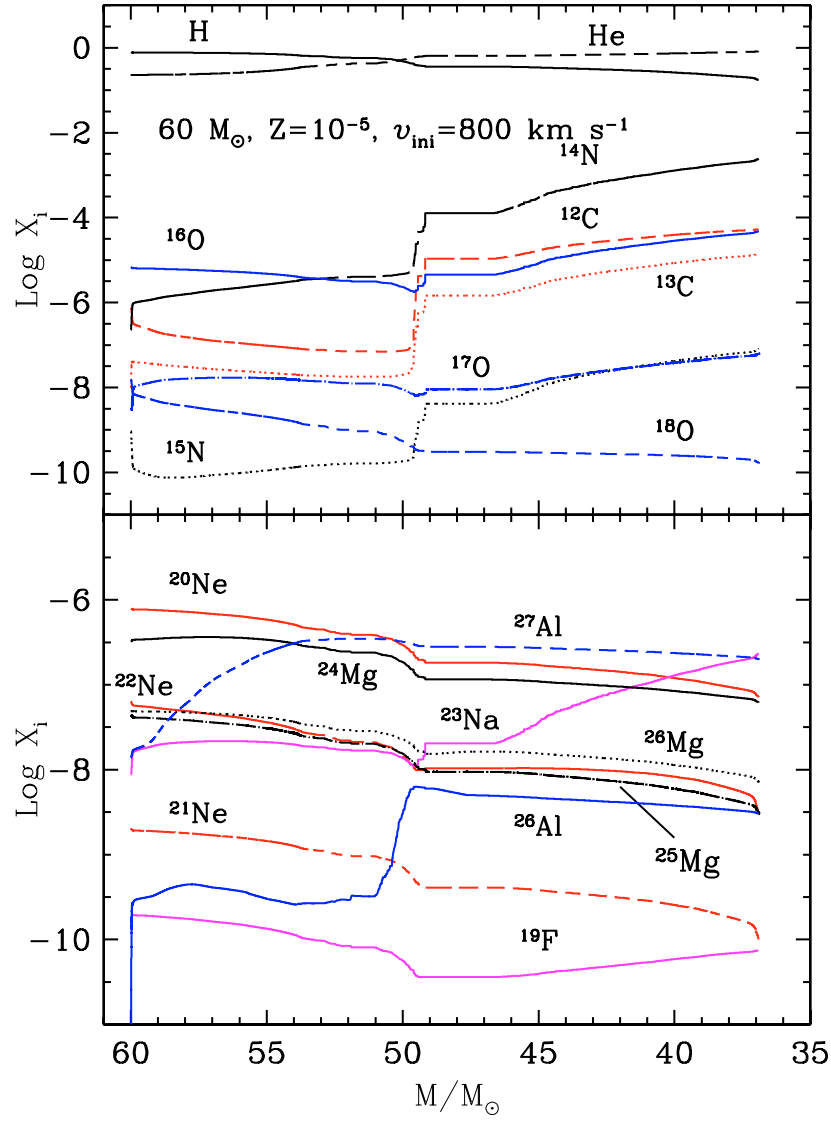

Fig. 1. Evolution as a function of the remaining mass of the surface abundances in mass fraction for a $60 M_{\odot}$ stellar model at $Z=10^{-5}$ and with $v_{\text {ini }}=800 \mathrm{~km} \mathrm{~s}^{-1}$, i.e. an initial ratio of angular velocity to the critical value $\Omega / \Omega_{\mathrm{c}}=0.85$.

the chemical elements from the $\mathrm{He}, \mathrm{C}, \mathrm{O}$ core into the H-burning shell.

- The mixing produces large surface enrichments in nitrogen and, depending on rotation, in other heavy elements like $\mathrm{C}$, $\mathrm{O}, \mathrm{Ne}$ and s-elements. This allows radiative stellar winds, so that low $Z$ stars experience mass loss during their AGB or red supergiant phases.

- If the star makes a blue excursion in the HR diagram after the red supergiant stage, the contraction of the convective envelope brings it to critical rotation and drives intense winds.

We examine the problem of $\omega$ Cen in relation with models of massive and AGB-stars. Some models were already calculated (Meynet et al. 2006) and we also give here a model of massive stars at $Z=10^{-5}$ for an intermediate rotation velocity. The results are shown in Figs. 1 and 2 and in Table 1.

Figure 1 shows the evolution of the surface abundances as a function of the remaining mass (which is equivalent to an age scale) for a model with an initial mass of $60 M_{\odot}$ and $Z=10^{-5}$. The initial fraction of the break-up angular velocity is $\Omega / \Omega_{\mathrm{c}}=0.85$, which corresponds to $v_{\text {ini }}=800 \mathrm{~km} \mathrm{~s}^{-1}$. (It is likely that the fraction of the break-up velocities is the meaningful quantity to consider rather than the $v \sin i$, since during star formation there is an enormous excess of angular momentum to be dissipated to allow the formation of star with 


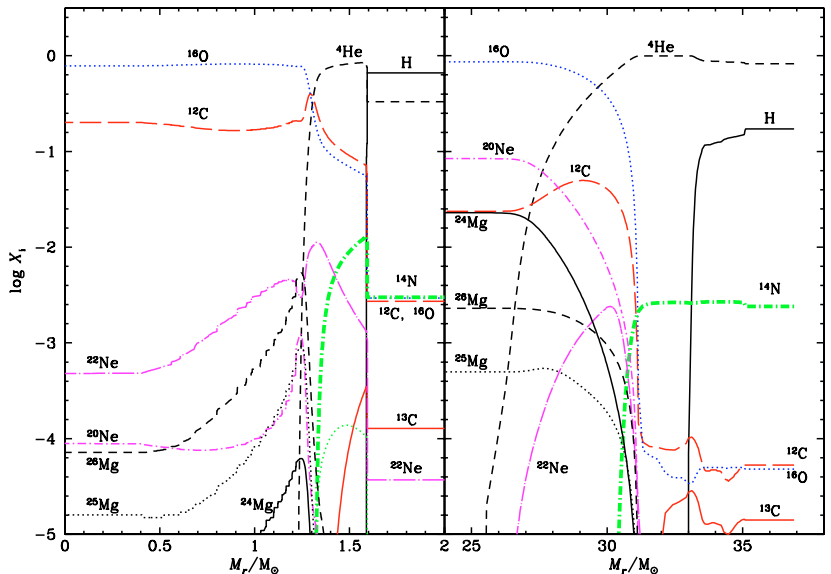

Fig. 2. Variations of the abundances (in mass fraction) as a function of the Lagrangian mass for (left) a $7 M_{\odot}$ stellar model in the early AGB phase, and for (right) a $60 M_{\odot}$ model at the end of the core C-burning phase. Both models were computed with $v_{\text {ini }}=800 \mathrm{~km} \mathrm{~s}^{-1}$ corresponding respectively to $\Omega / \Omega_{\mathrm{c}}=0.83$ and 0.85 . Both models have $Z=10^{-5}$.

sub critical velocities.) During a first phase, the actual mass decreases from 60 to about $51 M_{\odot}$. The changes of the surface abundances are due to rotational mixing of $\mathrm{CNO}$ processed material (mild decreases of ${ }^{12} \mathrm{C}$ and ${ }^{16} \mathrm{O}$ and increase of ${ }^{14} \mathrm{~N}$, the sum of CNO elements remaining constant during this phase). The mass lost, of about $9 M_{\odot}$, results from radiatively driven stellar winds and evolution at the break-up limit.

When the actual mass is $\sim 51 M_{\odot}$, the star is at the middle of the core He-burning phase ( $Y_{\mathrm{c}}$ equal to 0.45$)$ and with $\log T_{\text {eff }}=$ 3.850. An outer convective zone deepens in mass, dredgingup material to the surface. This produces sharp increases of the surface abundances in ${ }^{14} \mathrm{~N},{ }^{12} \mathrm{C},{ }^{13} \mathrm{C},{ }^{15} \mathrm{~N}$ as well as in $\mathrm{Na}$ and $\mathrm{Al}$. The enrichment in oxygen is modest. The amount of heavy elements increases up to more than 240 times the initial $Z$. From $\sim 50 M_{\odot}$, the star is essentially an He-star, corresponding to a Wolf-Rayet of type WN. A total of $12.20 M_{\odot}$ of helium is ejected, of which $5.86 M_{\odot}$ of newly synthesized helium (see Table 1).

Figure 2 compares the structures of an initial $7 M_{\odot}$ in the early AGB phase and of the previous $60 M_{\odot}$ model at the end of the central C-burning phase, the initial $\Omega / \Omega_{\mathrm{c}}=0.83$ and 0.85 respectively. The $7 M_{\odot}$ model, in contrast with the $60 M_{\odot}$ model, does not reach the break-up limit during the MS phase, however it shows more internal mixing. This is still visible in advanced stages (Fig. 2), where one notices milder composition gradients at the edge of the core in the AGB model of $7 M_{\odot}$ than in the late phases of the $60 M_{\odot}$ model. This results from the steeper internal $\Omega$-gradient in the 7 than in the $60 M_{\odot}$. The origin of this difference is the so-called Gratton-Öpik cell of meridional circulation, which brings angular momentum outward. This circulation term is weaker when density is higher (e.g. Maeder \& Meynet 2001), this is what happens in the 7 compared to the $60 M_{\odot}$ models at $Z=10^{-5}$ : less angular momentum is evacuated from the stellar center, henceforth steeper $\Omega$-gradients and more mixing.
Table 1. Comparison of the wind ejecta of a $60 M_{\odot}$ and a $7 M_{\odot}$ model with $Z=10^{-5}$ for different values of $\Omega_{\mathrm{c}} . \Delta Y$ and $\Delta Z$ are the amounts of new helium and heavy elements ejected. The brackets indicate the ratios in log scale of the abundances in the winds or in the AGB envelopes compared to the solar ratios.

\begin{tabular}{lccc}
\hline \hline$M$ & $60 M_{\odot}$ & $60 M_{\odot}$ & $7 M_{\odot}$ \\
$\Omega / \Omega_{\mathrm{c}}$ & 0.85 & 0.38 & 0.83 \\
& & & \\
\hline$\Delta Y$ in $M_{\odot}$ & 5.86 & 1.73 & 0.69 \\
$\Delta Z$ in $M_{\odot}$ & 0.09 & $2.6 \mathrm{e}-05$ & 0.12 \\
$\Delta Y / \Delta Z$ & 63.3 & $1 \mathrm{e}+05$ & 5.69 \\
{$[\mathrm{C} / \mathrm{Fe}]$} & 3.05 & -0.55 & 4.25 \\
{$[\mathrm{~N} / \mathrm{Fe}]$} & 4.36 & 1.79 & 4.70 \\
{$[\mathrm{O} / \mathrm{Fe}]$} & 2.92 & 0.33 & 3.77 \\
{$[\mathrm{~N} / \mathrm{C}]$} & 1.31 & 2.34 & 0.45 \\
{$[\mathrm{~N} / \mathrm{O}]$} & 1.44 & 1.43 & 0.93 \\
{$[\mathrm{Ne} / \mathrm{N}]$} & -3.90 & & -1.90 \\
{$[\mathrm{Ne} / \mathrm{Na}]$} & -0.80 & & 0.78 \\
{$[\mathrm{Na} / \mathrm{Mg}]$} & 1.00 & & 1.26 \\
{$[\mathrm{Mg} / \mathrm{Al}]$} & -0.90 & & 0.193 \\
${ }^{12} \mathrm{C} /{ }^{13} \mathrm{C}$ & 3.81 & 7.06 & 76.97 \\
${ }^{16} \mathrm{O} /{ }^{18} \mathrm{O}$ & 1183 & 2600 & 4200 \\
${ }^{17} \mathrm{O} /{ }^{18} \mathrm{O}$ & $2 \mathrm{e}+06$ & 19000 & 181 \\
\hline
\end{tabular}

The $7 M_{\odot}$ model at $Z=10^{-5}$ remains in the blue part of the HR diagram during the whole He-burning phase, preventing an outer convective zone to appear and to dredge-up the primary CNO elements. Only at the end of the core He-burning phase, it evolves to the red and approaches the base of the Asymptotic Giant Branch. An outer convective zone appears and produces an enormous enhancement of the surface CNO elements (cf. Fig. 2). In contrast, the weaker mixing of the $60 \mathrm{M}_{\odot}$ model does not bring oxygen and carbon to a level comparable to that of ${ }^{14} \mathrm{~N}$, which as shown by Fig. 1 keeps all the way higher. We also see the high helium content at the surface of the $60 M_{\odot}$ model as a result of mass loss and mixing, while the He-enhancement is modest in the $7 M_{\odot}$ AGB model.

The winds of the models considered are shown in Table 1, one notices the following features:

1. The winds of low $Z$ massive rotating stars eject large amounts $\Delta Y$ of new helium, while the envelopes of AGB, which is at the end entirely ejected, contain only a moderate amount of helium. $\Delta Y / \Delta Z$ of the order of $10^{2}$, or even more, are produced in massive stars.

2. There are large excesses of $\mathrm{N}$ ejected for both massive and AGB stars. The N-excess is larger for higher rotation.

3. Rotating AGB stars also have high productions of $\mathrm{C}$ and $\mathrm{O}$, about an order of magnitude below the $\mathrm{N}$-production.

4. The winds of massive stars show some $\mathrm{C}$ and $\mathrm{O}$ contributions, however even for fast rotation they are smaller than for $\mathrm{N}$ by one to two orders of magnitude. At moderate rotation, the production of $\mathrm{C}$ and $\mathrm{O}$ are negligible.

5. The winds of massive stars produce large amounts of ${ }^{13} \mathrm{C}$ and moderate amounts of $\mathrm{Na}$ and $\mathrm{Al}$. 

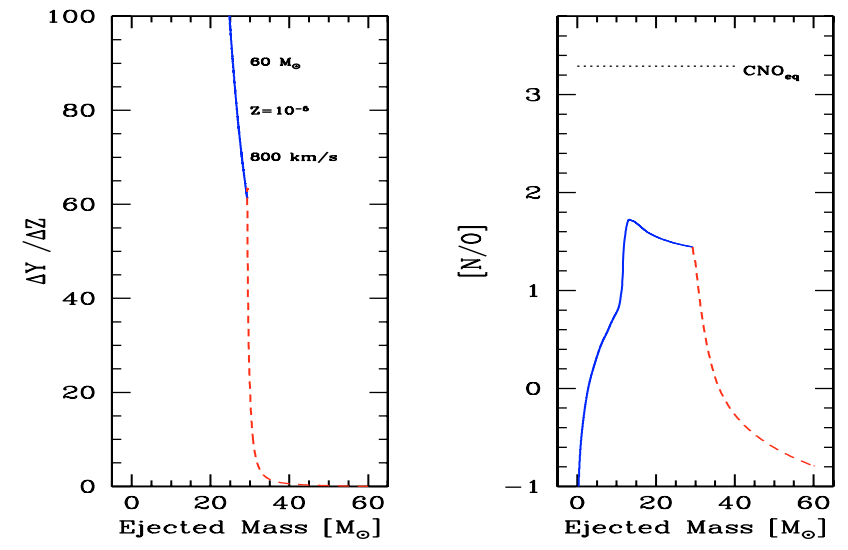

Fig. 3. Variations for a $60 M_{\odot}$ model with initial $\Omega / \Omega_{\mathrm{c}}=0.85$ and $Z=10^{-5}$ : left) the ratio $\Delta Y / \Delta Z$ as a function of the ejected mass; right) the same for the ratio of nitrogen to oxygen normalized to solar. The continuous line corresponds to the mass ejected by stellar winds, the dashed line shows how the ratios would vary if some amount of mass from the pre-SN model is added to the ejecta. The dotted lines indicate the $\mathrm{CNO}$ equilibrium values obtained in the convective core of the star, when the mass fraction of hydrogen at the center is 0.34 .

6. AGB winds produce large excesses of $\mathrm{F}, \mathrm{Ne}$ and $\mathrm{Na}$ (cf. also Meynet et al. 2006) and moderate ones in $\mathrm{Mg}$ and $\mathrm{Al}$.

7. The large excess of ${ }^{22} \mathrm{Ne}$ together with excesses of ${ }^{25} \mathrm{Mg}$ and ${ }^{26} \mathrm{Mg}$ in AGB stars imply a significant production of s-elements.

8. The production of s-elements in massive stars is possible if they lose enough mass to enter the WC stage.

There are evidently important uncertainties, such as the rotational velocities of the first stellar generations, the mass loss rates by stellar winds at low $Z$, the relative importance of the magnetic field, the distribution of stellar masses, etc.

Contrarily to simple expectations, AGB models exhibit nucleosynthetic products with signatures of higher temperatures than massive stars. In both cases, the synthesis is made by the $\mathrm{CNO}, \mathrm{NeNa}$ and $\mathrm{MgAl}$ cycles in the $\mathrm{H}$-shell burning, which receives some ${ }^{12} \mathrm{C}$ diffusing by rotational mixing out of the He-burning core. In AGB stars, most of the ejection occurs rather late in the nuclear evolution, i.e. near the end of the He-burning phase in the TP-AGB phase. In massive stars, most of the wind ejecta occurs earlier, from a synthesis at lower temperatures $T$ in the course of the He-burning phase. This explains the different $T$-signatures, which offer a powerful way to discriminate whether AGB or massive stars are the main source of the peculiar bMS abundances.

We examine how the ratios $\Delta Y / \Delta Z$ and, for example, $\mathrm{N} / \mathrm{O}$ (in mass fraction) vary as a function of time or of the mass ejected in the wind during the stellar lifetime. This is shown in Fig. 3 for the case of the fast rotating $60 M_{\odot}$ stellar model at $Z=10^{-5}$. At the beginning $\Delta Y / \Delta Z$ is not defined, then it tends to infinity when some newly synthesized helium appears at the surface without heavy elements. When the ejected mass approaches about $25 M_{\odot}, \Delta Y / \Delta Z \sim 100$, and at the end of the stellar lifetime $\Delta Y / \Delta Z \sim 63$ in the wind ejecta. The new heavy elements in the winds are in the form of new $\mathrm{CNO}$ elements, without new iron.

The dashed line in Fig. 3 shows how the addition of material from the pre-supernova (SN) model changes the ratios. As soon as some $\mathrm{SN}$ ejecta are present, much lower values of $\Delta Y / \Delta Z, \mathrm{~N} / \mathrm{C}$ and N/O are obtained. Even the addition of small amounts of mass ejected by SN changes a lot the ratios of the ejecta. This is a critical point, since one does not know the $\mathrm{SN}$ properties and nature of remnants at low $Z$. If black holes form, they likely swallow most of the $\alpha$-rich layers of the pre-SN. In this respect, the $\Delta Y / \Delta Z$ ratio offers a particularly sensitive test of the relative importance of wind and SN ejecta, the same is true for the ratio of $\mathrm{N}$ to $\alpha$-rich nuclei.

\section{Interpretation of the bMS properties}

We see from Table 1 that models of massive stars are able to produce high or even very high $\Delta Y / \Delta Z$ ratios as indicated by the bMS sequence of $\omega$ Cen, a moderate rotation producing higher ratios. The absence of a $\mathrm{C}$-excess points in favor of a not too extreme rotation, maybe of the order of $\Omega / \Omega_{\mathrm{c}}=0.5$. The observed $\mathrm{N}$-excess, which are not as extreme as in the wind of the fast rotating model, is also in better agreement with an average rotation of the indicated order. This is remarkably converging.

The comparisons point toward a source of the large helium production in massive stars, rather than in AGB stars. This is in agreement with the general view that stars of higher masses form at very low $Z$. Another clear conclusion is (cf. Fig. 3) that there is very little contribution to the yields from supernovae, otherwise $\Delta Y / \Delta Z$ would be strongly reduced. The above results do not exclude some contributions from AGB stars, which would not reduce too much the $\Delta Y / \Delta Z$ ratios and would make some contributions to s-elements, however as shown by Piotto et al. (2005) it is unlikely that AGB stars can produce enough helium. Another possible source of s-elements is in massive stars, if they lose enough mass to enter the WC stage. At this stage, the relative contributions of massive and AGB stars are unknown. We emphasize that further pertinent tests on this critical question can be provided by abundance ratios like $\mathrm{Mg} / \mathrm{Al}$, $\mathrm{Na} / \mathrm{Mg}, \mathrm{Ne} / \mathrm{N}$ as well as by isotopic ratios such as ${ }^{12} \mathrm{C} /{ }^{13} \mathrm{C}$, ${ }^{16} \mathrm{O} /{ }^{18} \mathrm{O}$ and ${ }^{17} \mathrm{O} /{ }^{18} \mathrm{O}$ which are $T$-sensitive (cf. Table 1 ).

The enrichment of $\omega$ Cen seems to result mainly from stellar winds, without the usual contributions in heavy elements from supernovae. What is the reason for that? One may envisage two possibilities, which are not mutually exclusive:

\section{A) The particular chemical history of $\omega$ Cen:}

$\omega$ Cen likely experienced a high intial rate of star formation and a fast initial chemical enrichment. This hypothesis is consistent with both the absence of extremely metal poor stars with $[\mathrm{Fe} / \mathrm{H}] \leq-2$ and the evidence by Cunha et al. (2002) of the absence of contributions from SNIa up to $[\mathrm{Fe} / \mathrm{H}]=-0.8$.

Now, the lowest mass stars of the bMS down to at least $0.2 M_{\odot}$, as observed in $\omega$ Cen, needs about $2 \times 10^{8}$ yr to be formed since the time they left the birthline (cf. Stahler \& Palla 2004). This implies that all stars with an initial mass above, 
say, about $4 M_{\odot}$ had the time to contribute to the cluster enrichment. The wind ejecta of supergiants and AGB, with velocities of less than a few $10^{2} \mathrm{~km} \mathrm{~s}^{-1}$, may not escape the cluster core. Since $\omega$ Cen is probably the stripped core of an ancient dwarf galaxy feeding the Milky Way (cf. Bekki \& Freeman 2003; Gnedin et al. 2002), the tidal effects were large enough to remove the external galactic layers. Thus, it would not be surprising that most supernova ejecta at velocity above $10^{4} \mathrm{~km} \mathrm{~s}^{-1}$ also escaped from the globular core (cf. also Norris 2004; Piotto et al. 2005). The mentioned absence of ejecta from SNIa, which may originate from stars initially less massive than $8 M_{\odot}$ may be an additional argument for the escape of the winds from supernovae.

B) The general dominance of enrichments by stellar winds at low Z:

Another attractive hypothesis is that for some range of low $Z$ values, the enrichment by stellar wind is generally dominant, with reduced contributions from the onion skin layers of heavy elements in supernovae of type II. This would be the case if the core collapse in supernova explosions lead to the formation of black holes, which swallow most of the heavy elements formed. This may occur at lower $Z$, since mass loss, although present, is not extreme, which thus leads to larger masses in pre-SN models, which favors black hole formation.

It is premature to choose between these two possibilities, which are not exclusive in the case of $\omega$ Cen. Further observations of abundance ratios in $\omega$ Cen and their comparison with more extended grids of models are the way to follow.

\section{Conclusions}

The wind contributions of low $Z$ massive rotating stars are able to produce the high $\Delta Y / \Delta Z$ observed in the bMS sequence in $\omega$ Cen. The observations tend to favour an origin of the high helium observed by contributions from massive stars of intermediate rotation velocities.
At this stage, it is not clear whether the dominance of wind contribution is a general feature of low $Z$ stars or whether the tidal evaporation experienced by $\omega$ Cen has enabled it to lose most of the supernovae ejecta, keeping the enrichment from stellar winds.

Some critical abundance and isotopic ratios may offer further signatures of the contributions of AGB and massive stars.

\section{References}

Anderson, J. 1997, Ph.D. Thesis, Univ. California, Berkeley Bedin, L., Piotto, G., Anderson, J., et al. 2004, ApJ, 605, L128 Bekki, K., \& Freeman, K. C. 2003, MNRAS, 346, L11

Bekki, K., \& Norris, J. E. 2005 [arXiv:astro-ph/0512385] Cunha, K., Smith, V. V., Suntzeff, N. B., et al. 2002, AJ, 124, 379

Gnedin, O. Y., Zhao, H., Pringle, J. E., et al. 2002, ApJ, 568, L23

Gratton, R. 2005, in From Liltium to Uranium: Elemental Tracers of Cosmic Evolution, ed. V. Hill, P. Francois, \& F. Primas, IAU Symp., 228, in press

Ideta, M., \& Makino, J. 2004, ApJ, 616, L107

Maeder, A. 1992, A\&A, 264, 105

Maeder, A., \& Meynet, G. 2000, A\&A, 361, 159 (Paper VI)

Maeder, A., \& Meynet, G. 2001, A\&A, 373, 555 (Paper VII)

McWilliam, A., \& Smecker-Hane, T. A. 2005, ApJ, 622, L29

Meynet, G., Ekstrom, S., \& Maeder, A. 2006, A\&A, 447, 623

Umeda, H., \& Nomoto, K. 2003, Nature, 422, 871

Norris, J. E. 2004, ApJ, 612, L25

Norris, J. E., \& Da Costa, G. S. 1995, ApJ, 447, 680

Pagel, B. E. J., Simonson, E. A., Terlevich, R. J., \& Edmunds, M. G. 1992, MNRAS, 255, 325

Piotto, G., Villanova, S., Bedin, L. R., et al. 2005, ApJ, 621, 777

Slarais, M., Riello, M., Cassisi, S., \& Piotto, G. 2004, A\&A, 420, 911

Stahler S. W., \& Palla, F. 2004, The Formation of Stars (Wiley Weinheim), 587

Thielemann, F. K., Nomoto, K., \& Hashimoto, M. 1996, ApJ, 460, 408 